\title{
Full thickness macular breaks in rhegmatogenous retinal detachment with peripheral retinal breaks
}

\author{
P Riordan-Eva, A H Chignell
}

\begin{abstract}
Between 1975 and 1989, 11 eyes were treated for retinal detachments with both peripheral and full thickness macular breaks. Long term retinal reattachment was dependent on successful closure of peripheral retinal breaks. Direct treatment of the macular break was necessary in only one case, which redetached 3 months after vitrectomy. Vitrectomy is not the initial treatment of choice for uncomplicated retinal detachments with both peripheral and full thickness macular breaks, unless required for the closure of the peripheral retinal breaks.
\end{abstract}

The relationship between macular breaks and rhegmatogenous retinal detachment is variable. In the highly myopic eye a break arising in the macular region may initiate rhegmatogenous retinal detachment, and in these cases subretinal fluid (SRF) accumulates around the break and extends towards the periphery. In other and usually more complex rhegmatogenous retinal detachments, such as complicating advanced proliferative vitreoretinopathy (PVR), in detachments secondary to vasoproliferative retinopathy, and in detachments associated with trauma, a macular break if present seems to play a secondary rather than an initiating role in the process of detachment.

In a third poorly documented but widely recognised group of apparently uncomplicated retinal detachments, a macular break develops in what had apparently been a normal macula, as a result of spread of SRF from a peripheral detachment initiated by a peripheral retinal break. This report describes our experience of 11 such cases.

\section{Materials and methods}

Among the retinal detachments treated between 1975 and 1989 by the Vitreo-Retinal Unit at St Thomas' Hospital, 11 cases of full thickness macular breaks arising secondary to peripheral retinal detachments have been identified. In all cases the break was identified as full thickness by preoperative and postoperative examination, as well as by direct peroperative identification in those eyes treated by vitrectomy. For the macular break to be considered secondary to spread of SRF from a peripheral detachment there had to be either; (i) a clear history of a progressive peripheral field defect, consistent with spread of SRF from identified peripheral retinal breaks (six cases); or (ii) a subtotal retinal detachment in which the SRF distribution was entirely consistent with spread from identified peripheral breaks, ' and in which there was no suggestion of the macular break preceding the peripheral break (five cases). All cases of macular breaks in which the temporal sequence of peripheral to central retinal detachment was not clearly established were excluded. Eyes with previously abnormal macular function as indicated by reduced vision or documented macular disease, myopia of more than 6 dioptres, history of recent trauma, evidence of vasoproliferative retinopathy, or PVR greater than grade B, were also excluded.

Patient details were obtained from hospital notes and the proforma sheets completed at the time of surgery, which form the basis of the Vitreo-Retinal Unit database. Each patient had undergone standard preoperative assessment, and retinal examination by indirect ophthalmoscopy with scleral indentation and slit-lamp biomicroscopy, including three mirror contact lens examination. Retinal findings had been recorded on standard colour coded retinal charts.

Eleven patients were identified, six male and five female. Ages ranged from 26 to 73 years with a mean age of 61 years. Six eyes were emmetropic and five were myopic (less than 6 dioptres). All eyes were phakic. The macular break retinal detachment was unilateral in all cases. The macular break was always a round hole at the fovea. The fellow eye was normal in nine, affected by mild atrophic macular degeneration in one, and had previously developed a rhegmatogenous retinal detachment without macular break formation in one.

\section{METHODS OF SURGERY}

During the collection of these cases, the surgical approach varied because:

(a) it was uncertain if the macular breaks were full thickness, and

(b) the contribution of full thickness macular breaks to rhegmatogenous retinal detachment was unclear.

Initially treatment was directed at closing the peripheral breaks using conventional methods; pars plana vitrectomy (PPV) was only undertaken when conventional surgery had failed to close them. Latterly, PPV was undertaken as a primary procedure if:

(a) it was thought necessary to drain all the SRF to achieve peripheral break closure, in which case this could be performed easily and safely via the macular break and supplemented by postoperative gas tamponade, or

(b) the peripheral breaks were too posterior for easy scleral buckling.

Conventional surgery and pneumatic retinopexy All eyes treated conventionally underwent 
cryotherapy to peripheral retinal breaks and then local scleral buckling, with or without encirclement, and with or without drainage of SRF. Macular buckling techniques were not used in any case.

Pneumatic retinopexy was performed by cryotherapy to the retinal breaks and then intravitreal injection of $\mathrm{C} 3 \mathrm{~F} 8$ gas, followed by appropriate posturing.

\section{Pars plana vitrectomy}

Pars plana vitrectomy (PPV) was performed using a standard triple port 20 gauge system. In all cases the schlieren phenomenon (direct visualisation of the passage of SRF into the vitreous cavity during aspiration in the vicinity of a retinal break) was used to confirm the presence of a full thickness break at the macula. In no case was it possible, either by direct visualisation or by tugging of the posterior detached retina during vitreous aspiration, to demonstrate vitreous attachment or traction in the macula area. Peripheral retinal breaks were treated with cryotherapy or endolaser photocoagulation. Scleral buckles were used when necessary. In all cases postoperative tamponade was accomplished with a $30 \%$ SF6/air mixture injected into the vitreous cavity after drainage of SRF via the macular break. Endolaser to the macular break was only used when specifically indicated.

The configuration of the initial detachment and the surgery undertaken are outlined in Table 1. Follow-up averages 27 months, with only two cases being followed up for less than 4 months from their last surgical procedure.

\section{Results}

In all 11 treated eyes the retina was successfully reattached. Final visual acuities ranged from $6 / 60$ to counting fingers.

Three eyes (cases 1-3) were treated entirely by conventional surgery, with a total of four procedures. The failure of initial surgery in case 3 was due to inadequate closure of the peripheral break. Among the three eyes (cases 4-6) undergoing vitrectomy after failure of conventional surgery, all the conventional procedures had failed due to missed peripheral breaks, but these could not be identified preoperatively and were found at vitrectomy. In the eye previously treated with two conventional procedures (case 6) endolaser was applied to the macular area at the time of vitrectomy. It was still felt that an open peripheral break was the reason for failure of conventional surgery and this was dealt with accordingly. The macular laser was only applied (probably unnecessarily) as a prophylactic measure against the possibility of redetachment from the macular break.

Among the five eyes treated by primary vitrectomy (cases 7-11), two had peripheral breaks too posterior to allow safe scleral buckling, and in the other three vitrectomy was performed to give access to the macular break for internal drainage of SRF. A total of nine procedures (eight vitrectomies and one pneumatic retinopexy) were required. Inadequate closure of the peripheral breaks was the reason for failure in two out of the three eyes requiring multiple procedures. In only one eye was failure of surgery due to redetachment from the macular break, and this did not occur until 3 months after the initial vitrectomy. It was successfully treated by redraining SRF via the macular break and endolaser to the macula, without any alteration in the scleral buckles.

\section{Discussion}

Although there is no previously published study specifically examining the management of rhegmatogenous retinal detachments with both macular and peripheral breaks in eyes without high myopia, preceding trauma, or significant proliferative vitreoretinopathy, the matter has been the source of much discussion. Some authors have felt that treating only the peripheral break is inadequate. ${ }^{2}$ whereas others have said

Table 1 Configuration of the initial retinal detachment and details of surgery undertaken

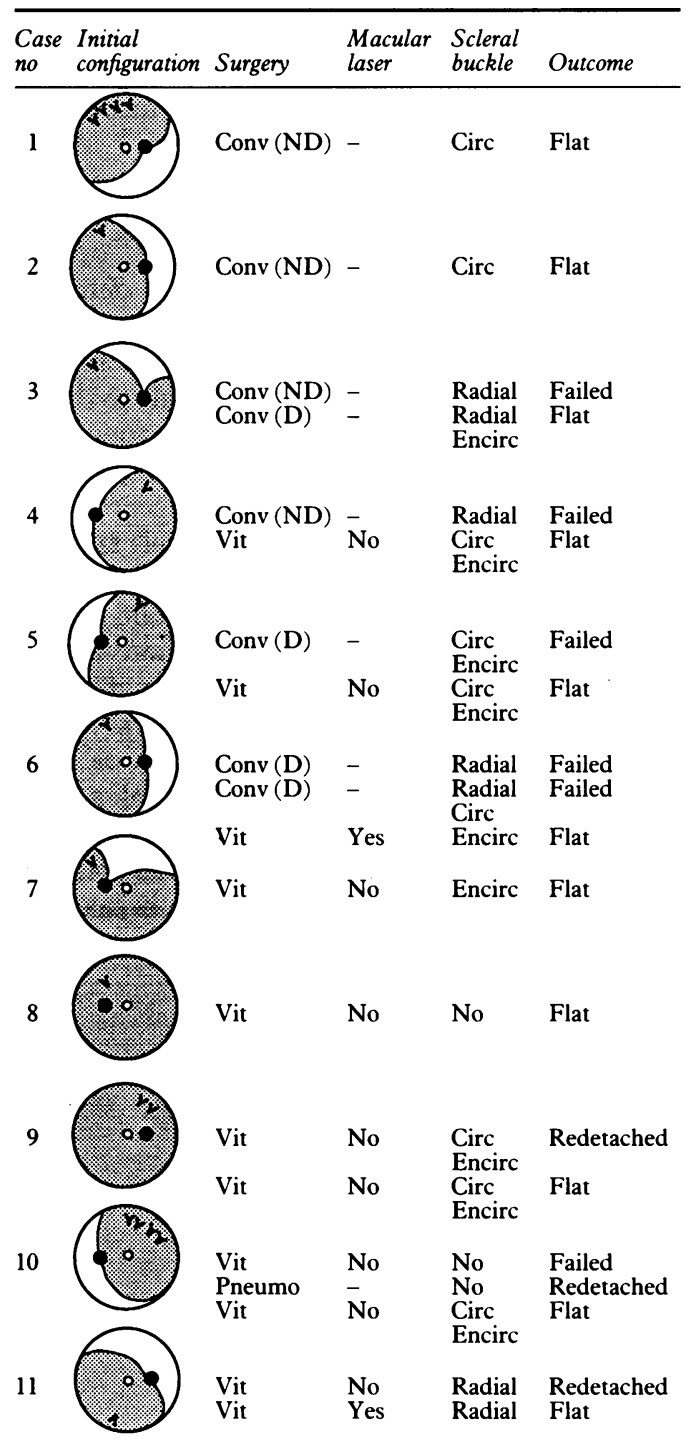

Conv $(\mathrm{ND})=$ conventional surgery without drainage of subretinal fluid

Conv $(D)=$ conventional surgery with drainage of subretinal fluid $\mathrm{Vit}=$ pars plana vitrectomy with internal drainage of subretinal fluid and intraocular gas tamponade

Pneumo=pneumatic retinopexy

Circ $=$ circumferential

Encirc $=$ encirclemen 
that it is usually sufficient and that the macular break only needs treatment if it is responsible for redetachment. ${ }^{3+}$ The matter is complicated by discussion of the validity of preoperative diagnosis of a full thickness macular break. Possible misdiagnosis was highlighted in a report of retinal detachments preoperatively thought to be associated with a full thickness macular break and treated by surgery to the peripheral break only. Of these as many as $75 \%$ had no features of a macular break following reattachment of the retina $^{5}$; such macular breaks have been termed pseudo-holes. ${ }^{6}$ The recovery of good vision after surgery casts doubt on the reliability of preoperative detection of full thickness macular breaks, although it has been reported. ${ }^{78}$

In our series the demonstration at PPV in eight cases that the macular breaks were indeed full thickness and transmitted SRF made us confident that careful preoperative examination had predicted accurately the presence of a full thickness break in all cases.

The operative findings at PPV gave no obvious clue as to the mechanism of formation of the macular break. There was no sign of vitreomacular attachment or traction, but the same operative techniques have demonstrated vitreous traction in macular hole retinal detachments in high myopes. ${ }^{9}$ Moreover, developments in the understanding of the pathogenesis of idiopathic macular holes have clearly shown that more sophisticated operative techniques may be required to detect vitreomacular adhesions. ${ }^{10}$ Macular break formation in the cases reported here may have represented an unusual response of the macula to the presence of underlying SRF, although in none of them was there any evidence of pre-existing macular disease.

The successful treatment of three cases by conventional surgery directed purely at the peripheral breaks, and six other cases by vitrectomy and gas tamponade without formation of a chorioretinal adhesion at the macula, demonstrates that macular breaks do not behave like peripheral breaks in the pathogenesis of rhegmatogenous retinal detachment. Whereas failure to seal a peripheral break in detached retina will always prevent retinal reattachment, the change in contour of SRF indicating the site of the open break, " the same rule does not apply to macular breaks. When a macular break develops in association with rhegmatogenous detachment from peripheral retinal breaks, it is virtually always only necessary to seal securely the peripheral breaks to achieve lasting retinal reattachment. In only one case in this series was it clearly established that the macular break was responsible for the reaccumulation of SRF after closure of the peripheral breaks, and that direct treatment of the macular break was then required (case 11). This eye had previously been vitrectomised. (Macular laser was used in case 6 as a questionable prophylactic measure only.)

Even in the presence of a full thickness macular break, the primary treatment of uncomplicated rhegmatogenous retinal detachments with peripheral breaks should be directed at closure of the peripheral breaks, which should in itself be enough to achieve lasting reattachment. PPV is useful to allow safe internal drainage of SRF, internal searching for peripheral breaks, postoperative gas tamponade, and closure of posterior breaks without scleral buckling, but there is no evidence that PPV for the sake of direct treatment of the macular break is necessary in the first instance.

1 Lincoff HA, Gieser R. Finding the retinal hole. Arch Ophthalmol 1971; 85: 565-9.

2 Muinos A, Mateus F, Hereida CD. Retinal detachment with holes in the posterior pole. Mod Probl Ophthalmol 1974; 12: 315-21.

3 Scott JD. Macular holes and retinal detachment. Trans Ophthalmol Soc UK 1974; 94: 319-23.

4 Theodossiadis GP. Letter to the editor. Graefes Arch Clin Exp Ophthalmol 1990; 228: 497-8.

5 von Pirquet SR. Treatment of macular breaks by photocoagulation. In: Schepens CL, Regan CDJ, eds. Boston: Little Brown, 1965: 178-82.

6 Meyer-Schwickerath G. Macular holes and retinal detachment. In: McPherson A, ed. New and controversial aspects of
retinal detachment. New York: $1968 ; 443-6$.

7 Binder S, Riss B. Advances in the intraocular techniques in the treatment of retinal detachments arising from holes of the
thes posterior pole. Brf Ophthalmol 1983; 67: 147-9.

8 Rashed O, Sheta S. Evaluation of the functional results after different techniques for treatment of retinal detachment due to macular holes. Graefes Arch Clin Exp Ophthalmol 1989; 227: 508-12.

9 Gonvers $M$, Machemer R. A new approach to treating retinal detachment with macular hole. Am $\mathcal{f}$ Ophthalmol 1982; 94 : 468-72.

10 Smiddy WE, Michels RG, Glaser BM, deBustros S. Vitrectomy for impending idiopathic macular holes. Am $\mathcal{F}$ Ophthalmol $1988 ; 105 ; 371-6$.

11 Lincoff $\mathrm{H}$, Kreissig I, Goldbaum M. Reasons for failure in non-drainage operations. Mod Probl Ophthalmol 1974; 12: $40-8$. 